\title{
Occult cardiac amyloidosis: the last chapter of a 2-year long story
}

\author{
Lucia Tattoli $^{1}$ (D) $\cdot$ Alessandro Dell'Erba $^{2} \cdot$ Giancarlo Di Vella $^{3} \cdot$ Eloisa Maselli $^{2}$
}

Accepted: 12 July 2019 / Published online: 31 July 2019

(C) Springer Science+Business Media, LLC, part of Springer Nature 2019

\begin{abstract}
Amyloidosis is characterized by deposition of insoluble fibrillar proteins in organs and tissues. The prognosis depends on where in the body amyloid deposition occurs, the amount of deposition, and the symptoms, which are often unspecific. Cardiac involvement is commonly associated with the immunoglobulin light-chain amyloidosis (AL) and may lead to sudden death. The case of a 53-year-old man's death due to unsuspected, undiagnosed AL predominantly involving the myocardium has been reported. His medical history had begun with gastroenterological symptoms. Angina pectoris and brain stroke occurred in the following years. He died after two years during the surgical implantation of a cardioverter-defibrillator because of cardiogenic shock. Post-mortem histologic examination revealed the presence of amyloid material in the lymph nodes, lungs, liver, spleen, kidneys, adrenal glands, stomach, and heart. The cause of death was cardiac failure secondary to primary systemic amyloidosis predominantly involving the heart. This case demonstrates that amyloidosis should be considered within the differential diagnoses and actively investigated in patients with unspecific and long-lasting symptoms; medical liability may also be suspected.
\end{abstract}

Keywords Systemic amyloidosis $\cdot$ Cardiac amyloidosis $\cdot$ Cardiomyopathy $\cdot$ Autopsy $\cdot$ Forensic pathology

\section{Case report}

In October 2014, a 53-year-old man started complaining about gastroenterological symptoms (abdominal pain and diarrhea) with severe weight loss $(>20 \mathrm{Kg})$. Abdominal examinations showed chronic colonic inflammation with hemorrhages, gastritis, and reflux esophagitis. In March 2015, an electrocardiogram (ECG) reflected a previous subepicardial ischemia. In April 2015, a treadmill stress test showed ST segment depression. In August 2015, he was admitted to the hospital with the diagnosis of colitis and chronic obstructive pulmonary disease. In September 2015, episodes of angina pectoris occurred,

Lucia Tattoli

luciatattoli@libero.it

1 S.C. Medicina Legale U, A.O.U. Città della Salute e della Scienza di Torino, Corso Bramante 88/90, 10126 Torino, Italy

2 Department of Interdisciplinary Medicine, Section of Legal and Forensic Medicine, University of Bari, Piazza Giulio Cesare 11, 70124 Bari, Italy

3 Department of Public Health and Pediatrics, Section of Legal Medicine, University of Turin, Corso Galileo Galilei 22, 10126 Torino, Italy with signs of biventricular hypertrophy and diastolic dysfunction at ECG and echocardiogram; coronarography revealed an intramural left anterior descending coronary artery. Upon discharge, diagnosis of angina abdominis was formulated. In March 2016, a brain stroke with left hemiparesis occurred; ECG showed low peripheral voltages with an increase of troponin; echocardiography showed bi-ventricular hypertrophy with pericardial effusion. A slight monoclonal gammopathy (IgG/Lambda immunoglobulins) was also found. In July 2016, the patient underwent bicameral implantable cardioverter defibrillator implantation because of progressive cardiac failure, but cardiogenic shock occurred, leading to death.

During autopsy, the heart weighed 450 g. Gross examination showed concentric ventricular hypertrophy (Fig. 1). Both kidneys (left weighing $100 \mathrm{~g}$ and right weighing $110 \mathrm{~g}$ ) were diffusely granular. The left and right lungs weighed 750 and $600 \mathrm{~g}$, respectively, with a massive right pleural effusion $(1650 \mathrm{cc})$. The remaining organs appeared grossly unremarkable.

Histologic examination demonstrated amorphous eosinophilic extracellular material suggestive of amyloid in the glomeruli, tubular interstitium, and arterial walls of both kidneys. In the heart, the same deposition was seen diffusely in the subendocardial layer and the walls of intramural arterial 


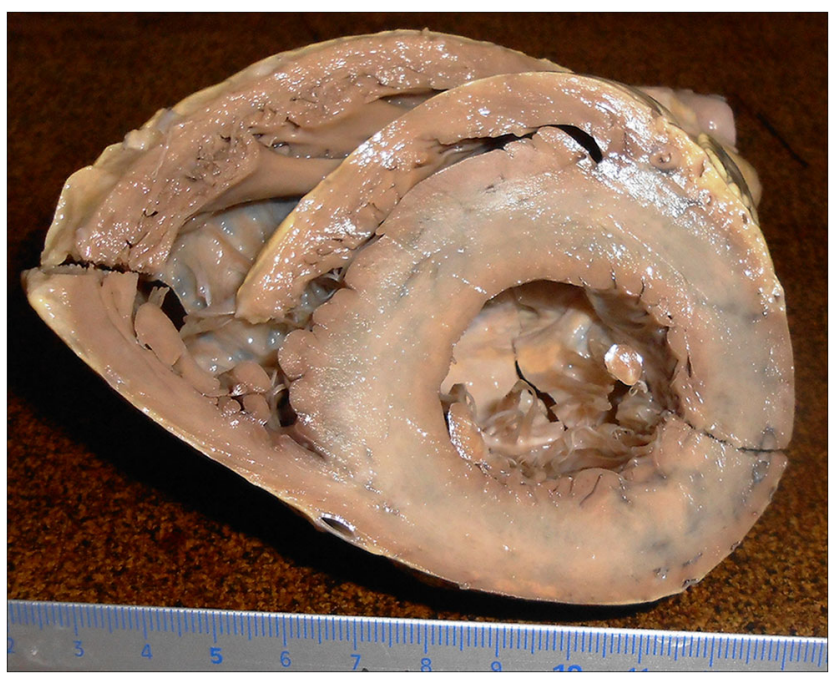

Fig. 1 Transverse section of the ventricles showing myocardial thickening

branches associated with myocyte atrophy (Fig. 2a-c). Vascular wall deposition of amyloid was also observed in the lymph nodes, lungs, liver (Fig. 3), spleen, pancreas, adrenal glands, and gastrointestinal tract. This material stained positive with Congo red; "applegreen" birefringence was also observed under polarized light. Sections of the myocardium demonstrated biventricular thickening as well as biatrial dilation and thickening of both atrioventricular valves.

Immunohistochemical analysis showed a predominance of lambda light chains within these cells (not shown).

These results were consistent with $\mathrm{AL}$ amyloidosis of the lambda light chain type. Death was caused by myocardial failure secondary to amyloid protein infiltration of the heart due to underlying primary systemic amyloidosis.

\section{Discussion}

Amyloidosis is a disease characterized by deposition of amyloid protein in organs and tissues throughout the body [1-3]. The amyloid deposition may be organ-specific or diffuse and multisystemic; infiltrated tissue exhibits damaged function by compression and atrophy of the interstitial cells [4]. Of the more than 20 forms identified, the three most common types are amyloid light chain (AL or primary systemic), amyloid associated (AA), and amyloid beta (Ab) $[1,5]$.

Cardiac amyloidosis is the most common infiltrative cardiomyopathy $[4,6]$. AL and transthyretin amyloidosis
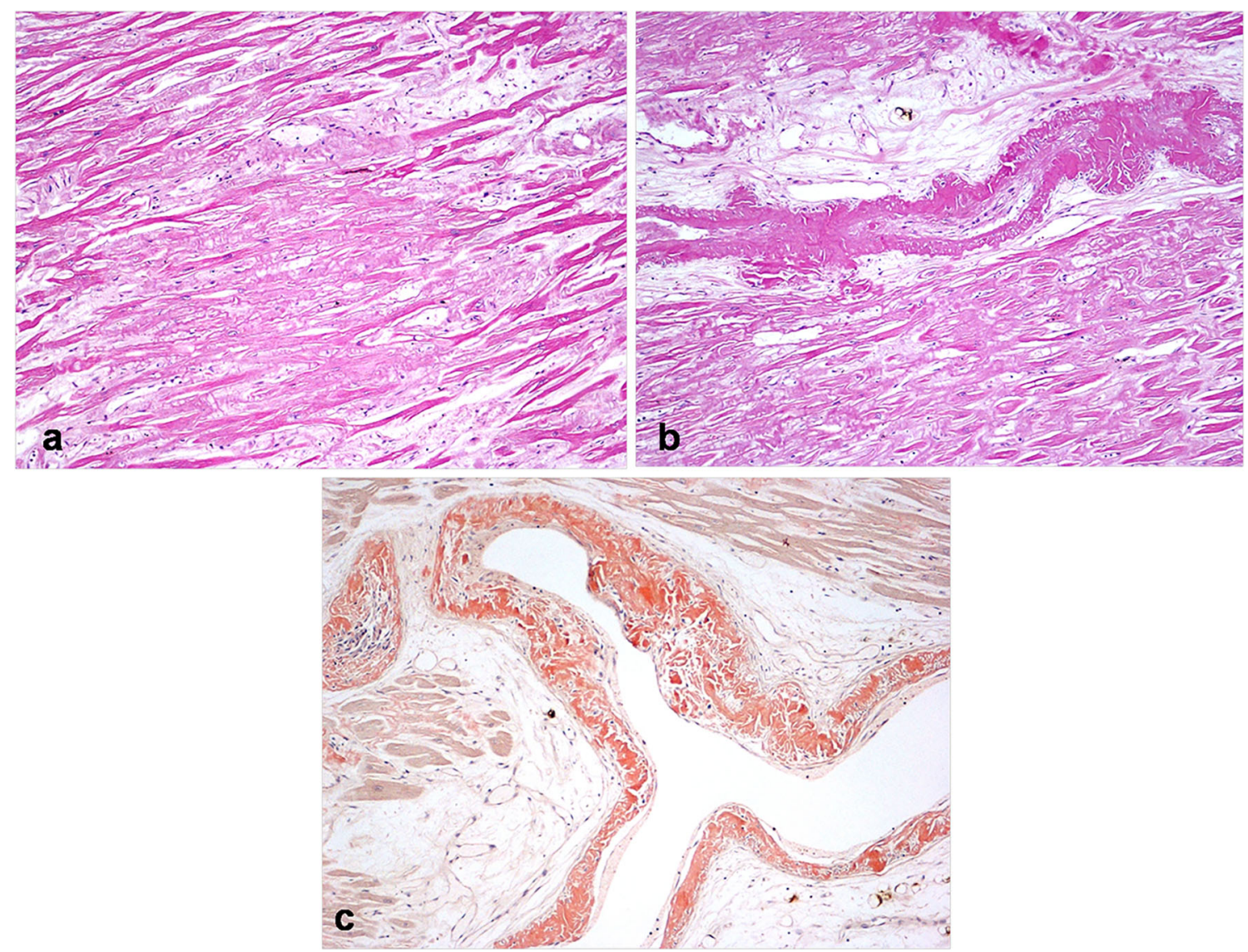

Fig. 2 Photomicrographs of heart section: massive subendocardial deposition of amorphous eosinophilic extracellular material (amyloid) with myocyte atrophy (a) as well as in the walls of intramural arterial

branches (hematoxylin\&eosin, magnification $\times 100)(\mathbf{b})$; positivity of the deposits in the vessels walls to the Congo Red staining (magnification $\times 100)(\mathbf{c})$ 


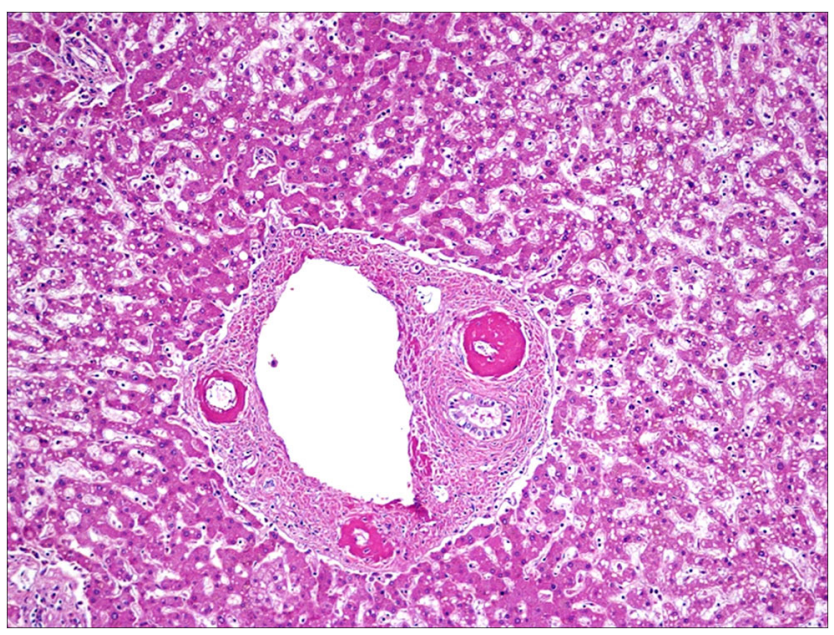

Fig. 3 Photomicrographs of liver section: amorphous extracellular material in the walls of the portal vessels (hematoxylin \& eosin, magnification $\times 100$ )

(ATTR) account for over 95\% of all cardiac amyloidosis [2]. Other amyloid types that can infiltrate the heart include amyloid A, apolipoprotein AI, heavy chain, and atrial natriuretic peptide (ANP) [2].

Cardiac involvement is characterized by diastolic cardiac failure and conduction system abnormalities. Angina or myocardial infarction is related to coronary artery amyloid deposition; involvement of the cardiac conduction system may lead to autonomic dysfunction and/or arrhythmias, which usually indicates a poor prognosis and may lead to sudden death $[2,3,7,8]$.

The most common presentation is a restrictive cardiomyopathy secondary to myocardial infiltration of amyloid protein that results primarily in diastolic dysfunction $[3,5]$.

The prognosis of amyloidosis is directly associated with the presence of cardiac involvement and depends on where in the body amyloid deposition occurs, the amount of deposition, and the onset of the symptoms, which are unspecific [5]. The overall median survival time following diagnosis is $<1$ year, ranging from 13 months to 4 months when the signs and symptoms of heart failure ensue $[4,8,9]$.

In the present case, the autopsy was fundamental to establish the diagnosis. The initial presentation was gastrointestinal involvement, with progressive worsening of cardiac function as biventricular hypertrophy at the ultrasound and ECG examination. At that time, the suspicion of infiltrative cardiomyopathy with systemic involvement (ECG anomalies, pericardial effusion, intestinal inflammation, and monoclonal gammopathy) may have arisen at least one year before the patient's death. For this reason, medical liability was initially alleged. Based on the clinical history, it could be supposed that amyloidosis was active from 2014 when considerable weight loss occurred. On the other hand, a differential diagnosis of infiltrative cardiomyopathy could have been made on the basis of clinical features and examination techniques in 2015 , one year before the death. Nevertheless, the patient was presented with the disease at a too advanced stage to benefit from curative treatments, with a low survival rate.

This case shows that amyloidosis should be considered within the differential diagnoses and actively investigated in patients with unspecific and long-lasting symptoms.

The cardiac amyloid disease has been referred to as one of the "great cardiac masqueraders" because its symptoms may mimic ischemic and valvular heart disease, restrictive cardiomyopathy, constrictive pericarditis, and ventricular arrhythmias [10].

This particular disease is not often considered a cause of disability or death until death occurs, disclosed by the forensic pathologist as medical examiner [11]. The forensic pathologist, as well as the hospital medical staff, must have in-depth knowledge and a practical experience of such cases to avoid misdiagnosis $[12,13]$. Additionally, a thorough autopsy on each patient with an unexplained or even seemingly apparent cause of death should be mandatory.

\section{Compliance with ethical standards}

Conflict of interest The authors declare that they have no conflict of interest.

Ethical approval This article does not contain any studies with human participants or animals performed by any of the authors.

\section{References}

1. Fikrle M, Palecek T, Kuchynka P, Nemeciek E, Bauerova L, Straub J, et al. Cardiac amyloidosis: a comprehensive review. Cor Vasa. 2013;55:60-75.

2. Donnelly JP, Hanna M. Cardiac amyloidosis: an update on diagnosis and treatment. Cleve Clin J Med. 2017;84:12-26.

3. Kyriakou P, Mouselimis D, Tsarouchas A, Rigopoulos A, Bakogiannis C, Noutsias M, et al. Diagnosis of cardiac amyloidosis: a systematic review on the role of imaging and biomarkers. BMC Cardiovasc Disord. 2018;18:221.

4. Morais GCP, Arruda MM, Bonadia JCA, Pozzan G. Cardiac amyloidosis: a challenging diagnosis. Autops Case Rep. 2014;4:9-17.

5. Morin J, Schreiber WE, Lee C. Sudden death due to undiagnosed primary amyloidosis. J Forensic Sci. 2013;58(Suppl 1):S250-2.

6. Desport E, Bridoux F, Sirac C, Delbes S, Bender S, Fernandez B, et al. Al amyloidosis. Orphanet J Rare Dis. 2012;7:54.

7. Mysorekar VV, Rao SG, Satish NT, Kamath SM. Cardiac amyloidosis: report of an autopsy case with review of the literature. Indian J Pathol Microbiol. 2010;53:842-3.

8. Grogan M, Dispenzieri A, Gertz MA. Light-chain cardiac amyloidosis: strategies to promote early diagnosis and cardiac response. Heart. 2017;103:1065-72.

9. Shah KB, Inoue Y, Mehra MR. Amyloidosis and the heart: a comprehensive review. Arch Intern Med. 2006;166:1805-13.

10. Petersen EC, Engel JA, Radio SJ, Canfield TM, McManus BM. The clinical problem of occult cardiac amyloidosis. Forensic implications. Am J Forensic Med Pathol. 1992;13:225-9.

11. Kemp W. Cardiac amyloidosis: a review of its causes and the morphologic spectrum found at forensic autopsy. Eur J Forensic Sci. 2014;1:1-4. 
12. Tattoli L, Krocker K, Sautter J, Tsokos M. Multidrug-related leukocytoclastic vasculitis raising suspicion of sexual homicide-things are not always what they seem. Int J Legal Med. 2016;130:179-83.

13. Tattoli L, Solarino B, Schiraldi O, Di Vella G. A case of lethal idiopathic plasmacytic lymphadenopathy with polyclonal hypergammaglobulinemia: a medical challenge for the forensic pathologist. J Forensic Sci. 2016;61:1139-42.

Publisher's note Springer Nature remains neutral with regard to jurisdictional claims in published maps and institutional affiliations. 